\title{
LOS MOVIMIENTOS SOCIALES Y LA ACCIÓN JUVENIL: apuntes para un debate*
}

\author{
María Isabel Domínguez ${ }^{* *}$
}

\begin{abstract}
Resumen: El trabajo aborda el rol que está jugando la juventud como generación, en el marco del aumento de las acciones colectivas y convivencia de movimientos sociales de múltiples orientaciones. Se toman en cuenta dos dimensiones: su peso en el marco de movimientos que involucran a distintos sujetos y cuyas reivindicaciones tienen un alcance más general, así como en qué medida, en tanto jóvenes, se convierten en actores sociales específicos, reconocidos (y autorreconocidos) como sujetos en sí mismos. Se constata que la juventud en esta etapa no ha constituido de manera significativa movimientos sociales con marcado carácter generacional y se formula la hipótesis de que el acelerado ritmo de los cambios que están teniendo lugar en nuestra época, imprimen un mayor dinamismo al contexto en que se configuran las generaciones y limitan las posibilidades de conformarse como tal, con una identidad colectiva definida, frente a otros criterios estructurantes de mayor estabilidad como el género o la etnia o frente a conflictos sociales más visibles y generales como la defensa de los derechos humanos o del medio ambiente. Sin embargo, la juventud constituye una relevante masa crítica dentro de movimientos sociales de diferente carácter, tiene particular protagonismo en algunos de ellos y su presencia resulta vital pues representan la perspectiva de futuro.
\end{abstract}

Palabras clave: juventud, identidad generacional, movimientos sociales, participación juvenil.

Ponencia preparada para la Mesa Redonda "Protestas, Acción Colectiva y Movimientos Sociales" a presentarse en el XXV Congreso de la Asociación Latinoamericana de Sociología (ALAS), del 22 al 26 de agosto de 2005, en Porto Alegre, Brasil.

** La autora es Dra. en Sociología, dedicada al estudio de la juventud y las generaciones. Se desempeña como Directora Científica del CIPS y es Profesora Titular Adjunta de la Universidad de la Habana, Centro de Investigaciones Psicológicas y Sociológicas (CIPS), La Habana, Cuba.

Artigo recebido em 5 mar. 2006 e aprovado em 13 maio 2006. 
Hablar de protestas, resistencias y movimientos sociales en el momento actual constituye un reto teórico, metodológico y práctico por la complejidad del entorno internacional y la diversidad de sujetos sociales y expresiones que asumen esas resistencias lo que lleva a (re)plantearse muchas de las preguntas que a lo largo de décadas se han hecho numerosos teóricos del tema y líderes de los propios movimientos.

No pretenderé describir la complejidad de ese contexto signado por la globalización que condiciona, entre otras cosas, como dice Hugo Zemelman que "el conflicto, entonces no es con actores nacionales sino con actores invisibles, ni siquiera con las grandes corporaciones sino con la clase ejecutiva de esas corporaciones transnacionales que tienen un discurso propio y con proyección histórica" (Zemelman, 2003, p. 281), lo que cuestiona la naturaleza real de los sistemas políticos y contribuye a la emergencia de múltiples tipos de sujetos, pero atomizados, cuyas resistencias unas veces no rebasan los marcos de propuestas subculturales o contraculturales ${ }^{1}$ $\mathrm{y}$, en otras, logran conformar movimientos sociales algunos de los cuales se orientan gradual o directamente a la política y otros no se plantean propósitos propiamente políticos sino culturales o societales en un sentido amplio. ${ }^{2}$

Lo que sí es incuestionable es que el siglo XXI se ha iniciado con un reforzamiento de las resistencias a escala planetaria y con evidentes expresiones de globalización de las mismas a partir de la convergencia en identificar al capitalismo y especialmente al neoliberalismo, como la principal fuente de problemas del mundo de hoy, en lo económico, social, cultural y ecológico y la búsqueda de nuevas alternativas de desarrollo ${ }^{3}$ (Amín y Houtart, 2003, p. 7).

En este marco de aumento de las acciones colectivas y convivencia de movimientos sociales de múltiples orientaciones, resulta de particular interés entender qué rol está jugando la juventud como generación. Ese análisis requeriría tener en cuenta al menos dos dimensiones. Por una parte, identificar el peso de la juventud en el marco de movimientos que involucran a distintos sujetos y cuyas 
reivindicaciones tienen un alcance más general y, de otra, conocer en qué medida, en tanto jóvenes, se convierten en actores sociales específicos, reconocidos (y autorreconocidos) como sujetos en sí mismos.

Para ello resulta de gran utilidad metodológica partir de la categoría sociológica generaciones y entender el significado de la identidad generacional como identidad colectiva.

Parto de considerar a las generaciones "como el conjunto histórico - concreto de personas, próximas por la edad y socializadas en un determinado momento de la evolución de la sociedad, lo que condiciona una actividad social común en etapas claves de formación de la personalidad que da lugar a rasgos estructurales y subjetivos similares que la dotan de una fisonomía propia" (Domínguez, 1994). ${ }^{4}$

$\mathrm{Si}$, a la vez, convenimos con A. Melucci que una identidad colectiva es "una definición interactiva y compartida, producida por varios individuos que interactúan y que hace referencia a las orientaciones de su acción, así como al ámbito de oportunidades y restricciones en el que tiene lugar la acción" (Melucci, 1989, p. 34), se aprecia con claridad el peso que puede tener el componente generacional en la formación de una identidad colectiva, de ahí las posibilidades latentes para dar lugar a la formación de movimientos sociales juveniles.

No cabe dudas que el referente por excelencia de la participación juvenil como protagonista de las resistencias antisistema se ubican en la década de los años 60s e inicios de los 70s, cuando su presencia fue relevante en hechos como la toma del poder político mediante la lucha armada que llevó al triunfo de la Revolución Cubana; como miembros de organizaciones de izquierda y partidos políticos, o a través del movimiento estudiantil que desembocó en el Mayo del 68 ; $u$ organizados en movimientos contraculturales como los hippies. En todos los casos cuestionaron la alienación de la sociedad capitalista, su modelo de consumo y sus posturas guerreristas. 
Aunque en esa época era posible ver a los jóvenes como participantes de movimientos intergeneracionales, uno de los signos distintivos fue el marcado carácter generacional de muchas de sus expresiones, no solo en la movilización política sino, sobre todo, en la producción simbólica que marcó a las sociedades como un todo. De esa etapa diría Sergio Balardini al prologar el libro "La participación social y política de los jóvenes en el horizonte del nuevo siglo": "La década del sesenta, idealizada por muchos... fue también, el tiempo de explosión y expansión de las subculturas juveniles: de los jóvenes entre el Che y el "submarino amarillo". Una década que navega entre la radicalización política y la contracultura. Alternativos, iracundos, militantes y radicales. La sociedad se moviliza y los jóvenes ocupan la primera línea” (Balardini, 2000, p. 8).

Explicar ese proceso requeriría un análisis - ya por demás realizado por diferentes autores - de las circunstancias internacionales económicas, sociales y políticas que condicionaron ese malestar social y permitieron su enfrentamiento, además de coyunturas concretas en cada uno de los contextos. Solo mencionaré que el liderazgo juvenil se vio favorecido por la llegada masiva a la etapa juvenil de los nacidos durante el boom demográfico posterior a la Segunda Guerra Mundial, cohortes que disfrutaron de un ensanchamiento de las posibilidades educativas y de cambios en las industrias culturales, proveedoras de mensajes homogeneizantes y estimuladores de una movilidad social ascendente.

A pesar del predominio de ese espíritu de promoción del "éxito social" visto fundamentalmente como posibilidades de consumir - o quizás por su existencia - esa generación joven, con una mayor socialización entre pares y con una particular relación con las posiciones más irreverentes y cuestionadoras del pensamiento social y la cultura, entraron en conflicto con las formas tradicionales de entender el mundo y se plantearon la ruptura con los obsoletos modelos del pasado en casi todos los ámbitos de la vida. Acerca de estas rupturas se ha señalado: "En los años 60 comienza a extenderse un clima de renovación de las costumbres, provocado por una larga onda con una influencia que superará las fronteras nacionales de todo el 
mundo, que alteró las formas tradicionales de concebir el cuerpo, de relacionarse con las instituciones, de comprender la familia, de concebir la autoridad" (Urresti, 2000, p. 183).

La segunda mitad de los años 70s y la década de los años 80s implicaron un cambio significativo en el contexto social internacional y fueron particularmente distintas para la región latinoamericana. La crisis económica internacional, los regímenes dictatoriales en varios países del área y el auge de los movimientos insurgentes de liberación nacional en Centroamérica, entre otros factores, marcaron diferentes formas de expresión de la resistencia juvenil según los distintos escenarios.

Desde la integración conformista de la juventud de las sociedades capitalistas desarrolladas o el crecimiento de expresiones anómicas; el surgimiento de movimientos subculturales o contraculturales como el movimiento de rock en la Argentina y otros países (Beltrán Fuentes, 1989); movimientos sociales estudiantiles por reivindicaciones escolares (Franco, 2000, p. 111); hasta el elevado peso y protagonismo de la juventud en las luchas de Nicaragua, El Salvador o Guatemala, constituyeron el panorama de ese período.

En ese marco comienzan a delinearse las características de la nueva etapa que algunos han denominado de fragmentación social y enfriamiento político: “... se trata de un contexto que se complejiza y rompe con las dinámicas de agregación de la población antes vigentes, dificultando la movilización política, hecho que deriva de una creciente fragmentación de intereses que disuelve las bases objetivas de la solidaridad social" (Urresti, 2000, p. 187).

Fue en el escenario de los años 90s, caracterizado - en sentido general - por la apatía y el pasotismo, ${ }^{5}$ que la juventud se orientó a la construcción de lo que algunos dieron en llamar "un privado armónico", donde el consumo material, el individualismo y el no pensar ni comprometerse constituyeron la norma, como reacción a la evidente frustración e impotencia de generaciones anteriores que no pudieron alcanzar cambios significativos en el orden social y como rechazo a sus formas tradicionales de hacer la política. 
Estudios realizados en esa década permitieron concluir que "los jóvenes europeos prefieren más que nada afiliarse a grupos de interés general - particularmente aquellos vinculados al deporte - y menos a aquellos que actúan de acuerdo a intereses específicos, como es el caso de los sindicatos, de los partidos políticos o las asociaciones de consumidores" (Bendit, 2000, p. 32).

Algo similar se constató en Latinoamérica. Estudios realizados en Chile encontraron que la mayor participación juvenil se daba en organizaciones deportivas, seguidas de organizaciones vecinales y comunitarias, del ámbito educacional y organizaciones religiosas, en ese orden (Sandoval, 2000, p. 155).

A pesar de ese retraimiento, las energías juveniles comenzaron a canalizarse en otras direcciones a través de movimientos ecologistas, culturales, comunitarios y, en algunos casos, de mayor compromiso social como la defensa de derechos humanos, la solidaridad y la ayuda contra la pobreza. De ahí que especialistas en el tema juvenil llamaran a considerar la participación de este sector de la sociedad no solo desde su relación de empoderamiento respecto del mundo adulto, sino a reconocer las formas propias de empoderamiento que construyen y las transformaciones que se han dado en la expresión de los contenidos de la participación juvenil (Krauskopf, 2000, p. 128).

El final del siglo XX y el inicio del actual han visto un renacimiento de las resistencias frente al capitalismo neoliberal y sus efectos de crecimiento de la pobreza y las desigualdades así como frente a la ofensiva guerrerista que hoy se vive nuevamente.

\section{¿Qué representan esas movilizaciones?}

Sin lugar a dudas, dos grandes impactos iniciales se derivan del resurgir de la protesta global, al dar cuenta de que en el mundo existen numerosas fuerzas sociales interesadas en el cambio. El primero de ellos ha sido mostrar al capital globalizado que también la resistencia y las luchas se pueden globalizar y que no ha llegado aun "el fin de la historia". El segundo impacto es que ha estimulado el 
optimismo y la confianza en el futuro y ha permitido tejer redes de comunicación entre esa diversidad de actores que gradualmente toman conciencia de que no están solos. El propio lema del Foro Social Mundial de que "Otro mundo es posible" así lo atestigua, a la vez que pensadores de la talla de Samir Amín y François Houtart han dicho “...el futuro será el producto de la interacción entre los movimientos sociales..." (Amín y Houtart, 2003, p. 9).

\section{Pero, ¿de qué tipo de movimientos hablamos?}

Se trata de articular movimientos sociales que se definan como antisistema, portadores de utopías, caracterizados por la búsqueda de alternativas, capaces de conquistar espacios públicos y articularse con lo político y de buscar las convergencias estratégicas en medio de tanta multiplicidad y atomización (Houtart, 2003, p. 296-297).

Ya se habla de nuevos movimientos sociales de proyección global o movimientos sociales globales que se definen como " un actor colectivo constituido por individuos que entienden tener intereses comunes y, por lo menos en lo que se refiere a una significativa parte de su existencia social, una identidad común’ (Scott, 1990, p. 6, citado por Serbín, 2005,p. 28) que, para promover estos intereses, tienen capacidad de movilización masiva o utilizan esta capacidad como un elemento de presión y que se distinguen de otros actores colectivos tales como partidos políticos, grupos de presión o asociaciones voluntarias" (Serbín, 2005, p. 28).

Se señala que estos movimientos sociales globales "con frecuencia trascienden las nociones tradicionales de política y de ciudadanía, en función de articular planteamientos culturales vinculados a valores y estilos de vida específicos y, eventualmente, conllevan planteamientos de ruptura radical con las diferentes dimensiones del proceso de globalización” (Serbín, 2005, p. 28).

De manera que en la actualidad se plantean interrogantes acerca de qué significa la nueva configuración que parecen presentar las protestas y los movimientos populares en la región latinoamericana, 
en términos de sujetos, formas de organización y de lucha, referencias programáticas y horizontes emancipatorios (Seoane, 2003, p. 18).

El significado de este renacimiento de los movimientos sociales con el inicio del nuevo siglo, es incuestionablemente positivo, pero una valoración objetiva y crítica de sus efectos (Amín y Houtart, 2003; Serbín 2005) permite identificar que:

- Se han concentrado en la deslegitimación del sistema imperante pero aun se ha avanzado poco en la definición de objetivos estratégicos y en la propuesta de nuevas alternativas. Hay que entender que ese vacío programático de los movimientos sociales, los trasciende. Asistimos a una época en que luego del derrumbe de las experiencias de sociedades alternativas al capitalismo, el pensamiento social no ha logrado articular nuevas propuestas que, sin que ello implique la construcción de "el modelo" con carácter único, se puedan definir objetivos estratégicos comunes y líneas de acción concertadas. Sin dudas, la reconstrucción de un nuevo paradigma lleva tiempo y será el resultado de la labor conjunta de intelectuales comprometidos (con particular peso de los cientistas sociales) y activistas de los movimientos, a través de sus mutuas experiencias de pensamiento y de acción. Aunque este proceso ha comenzado, aun no ha solidificado suficientemente.

- Junto a la multiplicación y ampliación de las resistencias, aun es fuerte su fragmentación, no solo por las reales separaciones geográficas y sociales y la falta de medios para enfrentarlas, sino por la todavía débil convergencia de intereses.

- Esa falta de convergencia es claramente visible entre actores del Norte y el Sur, lo que habla de dificultades para articular los intereses globales con los regionales y locales.

- La falta de objetivos claros lleva en ocasiones a que las movilizaciones se concentren en la forma y pierdan de vista 
los contenidos, por lo que a veces son solo expresiones folclóricas o culturales, desvirtuadas y ridiculizadas por los instrumentos del sistema, en particular los medios de comunicación.

- En la misma dirección anterior, en ocasiones las acciones se reducen a expresiones de violencia criminal o callejera que también los deslegitima.

Aunque estos son rasgos de los movimientos sociales globales en general, algunos de ellos, sobre todo los dos últimos se dan con más frecuencia entre la juventud.

Resulta interesante constatar que la juventud en esta etapa no ha constituido de manera significativa movimientos sociales con marcado carácter generacional como ocurre con los movimientos feministas o de indígenas, por ejemplo. Sin embargo, constituyen una relevante masa crítica dentro de movimientos sociales de diferente carácter y han tenido particular protagonismo en los movimientos antiglobalización, en las manifestaciones por el "NO" a la Guerra en Irak por solo citar algunos.

\section{¿Por qué ocurre así?}

Entender los movimientos juveniles en la actualidad requiere quizás más que nunca antes - analizarlos en sus contextos y comprender la compleja trama de relaciones entre lo global - regional - nacional - local, que los marca como generación.

Cuestiones que van desde las de orden demográfico y que conforman sustantivas diferencias en cuanto a la magnitud numérica del sector joven en la sociedad ya se trate de sociedades envejecidas como las europeas u otras aun jóvenes como las latinoamericanas; hasta posibilidades reales de integración social a través de oportunidades sociales de acceso a la educación y al empleo o no; o la ubicación familiar por encima o por debajo de la línea de pobreza dentro de un mismo país, para mencionar algunos elementos, 
condicionan una creciente heterogeneidad juvenil que es la base de su también fuerte heterogeneidad de intereses y demandas.

Pero, a la vez - también como nunca antes - como resultado de la globalización económica y cultural y de la expansión de las comunicaciones y sus nuevas formas como Internet, es posible establecer vínculos globales que dan lugar a representaciones simbólicas transnacionales, que contribuyen a conformar una identidad juvenil con muchos rasgos compartidos.

Es en esa dinámica entre lo común y lo diverso que se mueven las generaciones jóvenes hoy: a veces más parecidos a jóvenes de otros confines que a sus propios padres; a veces, por el contrario, viviendo situaciones similares a las de generaciones anteriores y percibiendo que su mundo no tiene nada que ver con el de otras juventudes.

Por ejemplo, estudios realizados en Cuba entre miembros de los grupos de edades considerados en el país como jóvenes, ${ }^{6}$ demostraron que el segmento de 25 a 30 años habla de "la juventud" refiriéndose a los de menor edad y no se incluyen, a la vez que fundamentan sustantivas diferencias en relación con ellos tanto en los contextos de socialización como en la definición de rasgos estructurales y subjetivos (Domínguez, 2002). En este caso no pueden obviarse los efectos de la situación internacional sobre la sociedad cubana en la década de los años 90s con el derrumbe del socialismo en Europa Oriental y el recrudecimiento de las acciones de hostilidad de Estados Unidos, que han impactado la realidad nacional y han provocado cambios en la situación del grupo juvenil.

Todo ello nos permitiría formular la hipótesis de que quizás el acelerado ritmo de los cambios que están teniendo lugar en nuestra época, imprimen un mayor dinamismo al contexto en que se configuran las generaciones y limitan las posibilidades de conformarse como tal, con una identidad colectiva definida, frente a otros criterios estructurantes de mayor estabilidad como el género o la etnia o frente 
a conflictos sociales más visibles y generales como la defensa de los derechos humanos o del medio ambiente.

La complejidad del momento actual implica una ruptura de la lógica lineal entre presente y futuro y de la dicotomía entre inclusión y exclusión, procesos que se entrelazan o superponen, lo que cambia la tradicional dialéctica entre ruptura y continuidad en la sucesión generacional y cuestiona la manera simplificada de concebir la etapa juvenil como preparación para la vida adulta. Ello hace que cada vez más la juventud perciba con escepticismo la posibilidad de generarse proyectos de vida inclusivos y confiar en las perspectivas de futuro, de ahí que nuevamente sus intereses se estén orientando a la construcción de utopías y la búsqueda de alternativas para la (re)construcción de una sociabilidad diferente. Pero aun este es un proceso que está resurgiendo, que se enfrenta con la fuerza que tienen los amplios sectores juveniles que se dejan someter por los sentidos impuestos de aspirar a incluirse en la lógica del consumo. De ahí que sea poco probable esperar una mayor presencia de movimientos juveniles con marcado carácter generacional y resulte más común encontrar a la juventud formando parte de movimientos más amplios.

El contexto latinoamericano es importante expresión de esa complejidad global. El hecho de constituir la región más desigual del planeta, unido a sus fuertes tradiciones reivindicativas, en medio del desencanto por el fracaso social de las llamadas transiciones democráticas, y ante las interrogantes que abre la llegada al poder de nuevos gobiernos elegidos en representación de intereses populares, la convierten en escenario propicio para la emergencia de nuevos movimientos sociales y que se interpele acerca del lugar y el papel en ellos de la juventud.

Procesos dados en esta región, algunos con más historia como la Revolución Cubana, otros más recientes como la Revolución Bolivariana o el Foro Social Mundial, constituyen importantes 
experiencias a tomar en consideración en el camino de la búsqueda de nuevas alternativas.

Comentaba el Maestro Pablo González Casanova que "La alternativa de una democracia universal que construya mallas de poder en expansión podrá darse desde algunos estados - nación como Cuba..." (González Casanova, 1998, p. 24).

Asimismo, sobre el Foro de Porto Alegre se ha dicho “... éramos muchos los que creíamos que allí se estaban sentando las bases de este futuro nuevo, que se estaba creando un nuevo sujeto, posible protagonista del cambio" (Botey, 2002, p. 39).

Sin dudas, en la combinación de experiencias de resistencia pasadas y presentes - al capitalismo globalizado y sus múltiples formas de expresión sobre grupos sociales diversos y sobre contextos regionales, nacionales y locales también diversos y en la convergencia de propósitos que permitan delinear una plataforma programática común, está la posibilidad de plantearnos una verdadera alternativa de cambio. En ese proceso los movimientos sociales constituyen cada vez más una de las principales fuerzas y, dentro de ellos, el componente juvenil resulta vital pues representan la perspectiva de futuro.

Pero también en ese proceso está nuestro incuestionable rol como científicos sociales pues, concordando con Melucci "El conocimiento social ya no sirve para alimentar el sueño de certezas finales... Los científicos sociales no pueden eludir su responsabilidad de definir un posible futuro" (Melucci, 1998, p. 381).

\section{A manera de síntesis:}

El análisis de la participación juvenil en el panorama social y político de los últimos años permite constatar que la juventud en esta etapa no ha constituido de manera significativa movimientos sociales con marcado carácter generacional como ha ocurrido con otros grupos sociales como los movimientos feministas o indigenistas, por ejemplo, pero ello no significa que no haya tenido una relevante presencia 
dentro de movimientos sociales de diferente carácter y un particular protagonismo en algunos de ellos.

Ese comportamiento permite formular la hipótesis de que el acelerado ritmo de los actuales cambios epocales, modifican más significativamente los contextos en que se configuran y socializan las generaciones, hacen menos estables sus procesos de conformación y más difusas sus identidades como tales, frente a otros criterios estructurantes de mayor estabilidad como el género o la etnia o frente a conflictos sociales más visibles y generales como la defensa de los derechos humanos o del medio ambiente.

Por ello, es la combinación de perspectivas pasadas y presentes, en que lo generacional juega un papel fundamental para aprovechar las experiencias acumuladas y sumarle las energías renovadoras de la juventud, que es posible delinear una plataforma programática común y plantearse verdaderas alternativas de cambio social. En ese proceso, los movimientos sociales constituyen cada vez más una de las principales fuerzas y, dentro de ellos, el componente juvenil resulta vital pues representa la perspectiva de futuro.

\section{Notas}

1 Desde ya hace varias décadas, A. Pizzorno alertaba acerca de las diferencias entre subculturas y movimientos sociales cuando planteaba: “... tanto los movimientos sociales como las subculturas se encuentran fuera de los canales convergentes que conducen hacia el Estado como normador de intereses dominantes y hacia el Estado como funcionario de la sociedad civil ... pero mientras el movimiento social propone otros valores, la subcultura acepta, o padece, los valores de la sociedad global, elaborando solo marginalmente otros, que les permitan aislar a sus miembros, al menos parcialmente, de las consecuencias de las relaciones que le son desfavorables..." (Pizzorno, 1973).

2 Una clasificación de la relación entre movimientos sociales y contexto político - social señala tres maneras: "Una primera es la visión incremental y gradualista, finalista: los movimientos sociales, más o menos espontáneos, más o menos acotados en un "movimiento popular" que logra aglutinarlos y darles dirección política para la transformación social. 
O sea, un proceso de gradual politización de los movimientos sociales. Una segunda visión toma en cuenta la crisis de las instituciones políticas tradicionales (especialmente los partidos y las representaciones parlamentarias), y descubre en los movimientos sociales las "nuevas formas de hacer la política", con actores sociales que se van definiendo de manera novedosa: los jóvenes, los inmigrantes, las mujeres. El tema en cuestión, en este caso, es como los nuevos actores se vinculan con las viejas estructuras. En tercer lugar podemos mencionar una visión más "culturalista" y societal: estos movimientos sociales nuevos no deben ser interpretados en clave política (si por esto se entiende una lucha por el poder), sino como prácticas centradas en la construcción de identidades colectivas y de reconocimiento de espacios de relaciones sociales" (Jelín, 1985, p. 14).

3 Davos (1999), Seatle (1999), Génova (2001), el Forum Social Mundial en Porto Alegre (2002, 2003 y 2005) y la India (2004), son algunas de las más significativas expresiones de la movilización antiglobalización.

4 Esta definición - aunque coincide con la mayor parte de las que han sido elaboradas a lo largo de la historia del concepto al destacar que es un grupo de personas que vive y se forma en una determinada etapa histórica - pone el énfasis en el carácter de la actividad social desplegada colectivamente como lo realmente conformador de la generación.

5 Término acuñado en los años 80s para caracterizar la conducta de la juventud expresada como un "yo paso" ante las elecciones, la afiliación política y otras acciones participativas.

6 En Cuba se consideran jóvenes las personas comprendidas entre 14 y 30 años, por estimarse que en ese período tienen lugar un conjunto de procesos de orden biológico, psicológico y social que los definen como tales y porque las políticas de juventud abarcan ese intervalo (Domínguez, 2003, p. 67).

Social movements and youth's participation: notes for a debate

\begin{abstract}
This article has the purpose of contributing to the understanding of the role that is played by the generation of youth, in the framework of the increase of the collective actions and coexistence of social movements of multiple orientations, taking into account two dimensions: youth's weight in movements that involve different subjects and whose claims have a more general reach, and
\end{abstract}


in what measure they become converted into specific social actors, recognized and self-recognized as subjects themselves. The article verifies that in this stage, the youth has not yet constituted significant social movements with specific generational character, and a hypothesis is formulated about the quick rhythm of the changes that are taking place in our time, pointing to a greater dynamism of the context in which these generations are configured. Such dynamism limits the possibilities of conformity to a defined collective identity, in contrast with other more stable approaches such as gender or ethnics belonging to, or in the face of, more visible and more general social conflicts like the defence of human rights or the environment. However, youth constitutes an outstanding critical mass inside social movements of different character. They have a particular protagonistic role in some of them, and their presence is vital because they represent the future perspective.

Key-words: youth, generational identity, social movement, youth's participation.

\section{Referencias bibliográficas}

AMÍN, Samir. La amplitud de los desafíos: reflexiones sobre los orígenes y el comportamiento de las resistencias y las luchas: la dimensión cultural y la dimensión política. In: AMÍN, S.; HOUTART, F. (Eds). Globalización de las resistencias: el estado de las luchas 2003. Barcelona: Icaria, Antrazit, Caritas Española, 2003. p. 305-332.

AMÍN, Samir; HOUTART, François. Introducción. In: AMÍN, S.; HOUTART, F. (Eds). Globalización de las resistencias: el estado de las luchas 2003. Barcelona: Icaria, Antrazit, Caritas Española, 2003. p. 7-10.

BALARDINI, Sergio. Prólogo. In: BALARDINI, S. (Comp.). La participación social y política de los jóvenes en el horizonte del nuevo siglo. Buenos Aires: CLACSO, 2000. p. 7-18.

BELTRÁN FUENTES, Alfredo. La ideología antiautoritaria del rock nacional. Buenos Aires: Centro Editor de América Latina, 1989.

BENDIT, René. Participación social y política de los jóvenes en países de la unión Europea. In: BALARDINI, S. (Comp.). La participación social y política de los jóvenes en el horizonte del nuevo siglo. Buenos Aires: CLACSO, 2000. p. 19-57. 
BOTEY, Jaume. Porto Alegre, otro mundo posible. In: MONEREO, M.; RIERA, M. (Eds). Porto Alegre: otro mundo es posible. Madrid: El Viejo Topo, 2002. 27 - 40

DOMÍNGUEZ, María Isabel. Las generaciones y la juventud: una reflexión sobre la sociedad cubana actual. La Habana: Centro de Investigaciones Psicológicas y Sociológicas (CIPS), 1994. (Tesis doctoral).

Subjetividad juvenil en Cuba. La Habana: Informe de Investigación, Fondos del CIPS, 2002.

Juventud cubana y participación social: desafíos de una nueva época. In: DOMÍNGUEZ, M. I. (Comp.). La sociedad cubana: retos y transformaciones. La Habana: Editorial de Ciencias Sociales, 2003.

FRANCO, Bolívar. Centroamérica y Panamá: movimientos sociales juveniles y proyecciones hacia el nuevo siglo. Elementos para el debate. In: BALARDINI, S. (Comp.). La participación social y política de los jóvenes en el horizonte del nuevo siglo. Buenos Aires: CLACSO, 2000. p. 109-118.

GONZÁLEZ CASANOVA, Pablo. La democracia de todos. In: SADER, E. (Ed.). Democracia sin exclusiones ni excluidos. Caracas: Nueva Sociedad, 1998. p. 23-33.

HOUTART, François. La amplitud de los desafíos: reflexiones sobre los orígenes y el comportamiento de las resistencias y las luchas. La dimensión social. In: AMÍN, S.; HOUTART, F. (Eds.). Globalización de las resistencias: el estado de las luchas 2003. Barcelona: Icaria, Antrazit, Caritas Española, 2003. p. 287-304.

. Los proyectos y los niveles de las alternativas. In: AMÍN, S.; HOUTART, F. (Eds.). Globalización de las resistencias: el estado de las luchas 2003. Barcelona: Icaria, Antrazit, Caritas Española, 2003. p. 353-368.

JELÍN, Elizabeth. Los movimientos sociales en la Argentina contemporánea: Una introducción a su estudio. In: JELÍN, E. (Comp.). Los nuevos movimientos sociales. Buenos Aires: Centro Editor de América Latina, 1985. p. 13-40.

KRAUSKOPF, Dina. Dimensiones críticas en la participación social de las juventudes. In: BALARDINI, S. (Comp.). La participación social y política de los jóvenes en el horizonte del nuevo siglo. Buenos Aires: CLACSO, 2000. p. 119-134. 
MEDINA CARRASCO, Gabriel. La vida se vive en todos lados. La apropiación juvenil de los espacios institucionales. In: MEDINA CARRASCO, Gabriel (Comp.). Aproximaciones a la diversidad juvenil. México, DF: El Colegio de México, 2000. p. 79-115.

MELUCCI, Alberto. Nomads of the present: social movement and individual needs in contemporary society. London: Hutchinson Radius, 1989. . La experiencia individual y los temas globales en una sociedad planetaria. In: IBARRA, P.; TEJERINA, B. (Edit.). Los movimientos sociales: transformaciones políticas y cambio cultural. Madrid: Editorial Trotta, 1998. p. 361-381.

PIZZORNO, Alejandro. Introducción al estudio de la participación política. Buenos Aires: SIAP, 1973.

SANDOVAL, Mario. La relación entre los cambios culturales de fines de siglo y la participación social y política de los jóvenes. . In: BALARDINI, S. (Comp.). La participación social y política de los jóvenes en el horizonte del nuevo siglo. Buenos Aires: CLACSO, 2000. p. 147-164.

SEOANE, José. Introducción. In: SEOANE, J. (Comp.). Movimientos sociales y conflicto en América Latina. Buenos Aires: OSAL/CLACSO, 2003. p. 11-19.

SERBÍN, Andrés. Globalización, regionalismo y sociedad civil. Caracas: CRIES, 2005. (Documento CRIES, n. 1).

TEJERINA, Benjamín. Los movimientos sociales y la acción colectiva: de la producción simbólica al cambio de valores. In: IBARRA, P.; TEJERINA, B. (Edit.). Los movimientos sociales: transformaciones políticas y cambio cultural. Madrid: Editorial Trotta, 1998. p. 111138.

URRESTI, Marcelo. Paradigmas de participación juvenil: un balance histórico. In: BALARDINI, S. (Comp.). La participación social y política de los jóvenes en el horizonte del nuevo siglo. Buenos Aires: CLACSO, 2000. p. 177-206.

ZEMELMAN, Hugo. Hacia una estrategia de análisis coyuntural. In: SEOANE, J. (Comp.). Movimientos sociales y conflicto en América Latina. Buenos Aires: OSAL/CLACSO, 2003. p. 279-285. 